\title{
Gynostemma pentaphyllum Supplement
}

National Cancer Institute

\section{Source}

National Cancer Institute. Gynostemma pentaphyllum Supplement. NCI Thesaurus. Code C117275.

A traditional Chinese medicine ( $\mathrm{TCM}$ ) derived from the plant Gynostemma pentaphyllum, an herbaceous climbing vine found in southern China and Southeast Asia, with potential antioxidant activity. Gynostemma pentaphyllum intake has also been used for its adaptogenic effect, to decrease cholesterol and high blood pressure, to strengthen the immune system, and to increase stamina and to improve memory. 